\title{
Register rerum ad Vol. 22
}

Fecit Wilhelm Baumann, Zurich

Allopurinol, prophylaxis of uric acid

lithiasis, 506 Ampicillin concentration in prostatic

tissue, 453 Amyloidosis, acute renal failure, 390 Anurie bei Nierenblutung wegen Ge-

rinnungsstörung, 399

Bladder, enlargment and substitution, 84

-, rectal, 1, 29, 47

Blasenhals, Suspension bei Inkontinenz,

185 Blasenhalsplastik bei Prostatektomie,

227 Blasenulkus nach Radiumbestrahlung,

429

Calcio urinario, Tirocalcitonina, 285 Cálculos urinarios, composición, 178 Calculs d'acide urique, 193, 492, 506

, analyse de la structure, 193

Calculs rénaux, classement des porteurs,

473

, dissolution chimique, 155

Carcinome du col, ulcère radique, 429 Cateterismo permanente ureteral, 366 Catheterization by paraplegic patients,

236 Cervical cancer, vesical ulcer following

$\mathrm{X}$-irradiation, 429 Circulación renal después de la nefrec-

tomia, 250 Colostomia analis, 29, 47 Congenital ureterectasis, study of the

nerve fibres, 414 Corticoides, prevención de las estre-

checes de las vias urinarias, 215 Cystectomia totalis, 1, 29, 47, 332, 435,

446 Cystectomy, ureters in the prevesical

space, 332

Dysglobulinemia, acute renal failure, 390

Ersatzblase, Absorption der Harnbe-

standteile, 435 Extravasación peripélvíca, 342

Fibrinolytische Aktivität des Harnes, 208

Fistulas y defectos de la uretra, 461

Harnblase, Erweiterung und Ersatz, 84 Hamleiter-Darm-Implantation, Sigma-

myotomie, 446 Harnröhre, Fisteln und Defekte, 461 Harnsäuresteinbildung, 193, 492, 506

Harnsäuresteine, 193 Harnstoffanstieg nach Prostataopera-

tionen, 312 Harnverhaltung bei Herpes zoster, 222 Hématurie, activité fibrinolytique de

Гurine, 208 Hemorrhagic diathesis, hematuria with

anuria, 399 Hémorrhagie rénale due à des troubles

de coagulation, 399 Herpes zoster, causa de retención

urinaria, 222 Hiperparatiroidismo primario con 
urolitiasis, 517 Hodentumoren bei eineiigen Zwillingen, 353 Hypercalcaemia, Tyreocalcitonin, 285 Hyperparathyroidisme primaire et lithiase urinaire, 517 Hypertrophie de la prostate, culture

bactérienne, 167

Implantación uretero-intestinal, sigma-

miotomia, 446 Incontinence à $\Gamma$ efíbrt, Marshall-

Marchetti, 324, 185 Insuffisance rénale aigue lors de dys-

globulinémie, 390

Intestinal loops, absorption of urine products, 435

Jumeaux, tumeurs testiculaires, 353

155

Kidney, intrarenal pressure, lí -, Mannitol in ischemia, 381 -, methods of preservation, 258 -, salt and water levels, 533

Lithiase urinaire et hyperparathyroidisme primaire, 517 Lithiasis, extravasation pélvienne, 342

Litiásicos, clasificación, 473

Index

rerum

555

Litiasis renal, quimiolisis, 155 - urica, análisis estructural, 193 Litogénesis urica, 193, 492, 506 Mannítol bei ischämisch geschädigten

Rattennieren, 381 Marshall-Marchetti, 185, 324 Meatoplastia, 468 Mégauretères, etude des

fibres nerveu-

ses, 414 Métabolisme du sel et de $\Gamma$ eau du

malade renal, 532 Multiple myeloma, acute renal failure,

390 Nephrectomy, renal blood perfusion, 250 Nephritis chronica interstitialis, 68 Nephrolithiasis, classification of individuals, 473 -, dissolutio, 155 -, peripelvic extravasation, 342 -, Thyreocalcitonin, 285 -, X-ray analysis, 193 Niere, Blutung bei Gerinnungsstörung, 399 -, Durchblutung nach einseitiger

Nephrektomie, 250 -, intrarenale Druckverhältnisse, 155 -, Konservierungsverfahren, 258 -, Mannitol bei Ischämie, 381 -, Salz- und Wasserhaushalt, 532 -, Tumoren bei tuberöser Hirnsklerose,

406 -, Versagen bei Dysglobulinämien, 390 Nierensteinchemolyse, 155 Nierensteinträger, Klassifizierung, 473

Orina, actividad fibrinolitica, 208

Paraplegic patients, intermittent

catheterization, 236 Peripelvine Extravasation bei Ureter-

steinen, 342 Phäochromocytom, Blutdruckkontrolle

nach Entfernung, 301 Pheochromocytoma, blood pressure

control after the removal, 301 Phosphothérapie de la lithiase urinaire,

483, 506 Plastie du col et prostatectomie, 227 Plastic surgery on urinary tract, 215 Prostata,

Harnstoffanstieg nach Opera-

tionen, 312 Prostataadenom, Bakteriurie, 167 Prostatagewebe, Ampicillin-Konzentra-

tion, 453

Prostatectomy with vesical neck plasty,

227 Prostatic hyperplasia, bacteriuria, 167 Prostatic operations, rise of blood urea. 
312 Pyelonephritis xanthogranulomatosis, 68

Reflux vésico-urétéral chez Гadult, 124 Reflux vésico-urétéral chez l'enfant, 97,

141 Rein, circulation après néphrectomie,

250 -, examen microscopique, 258 -, la pression intra-pyélique, 155 -, Mannitol et lesions

ischémiques, 381 -, métabolisme du sel et de Гeau, 532 Rektumblase, 1, 29, 47 Renal calculosis, chemical dissolution,

155

disease, salt and water levels, 532

leiomyosarcoma and tuberous sclerosis, 406

Retention urinaire et herpes zoster, 222 Retropubic suspension, 185, 324 Riñón, conservación, 258 -, insuficiencia aguda en las disglobu-

linemias, 390 -, la pression intrarenal, 155 -, Manitol en las lesiones isquémicas,

381 -, metabolismo hidrico y mineral, 532

Salz- und Wasserhaushalt des Nieren-kranken, 532

Sclérose cérébrale tubéreuse et tumeurs rénales, 406

Sondages vésicaux chez les paraplégi-ques, 236

Sondes urétérales de longue durée, 366

Stress incontinence, retropubic suspension, 185, 324

Strikturen nach plastischen Operatio-nen am Harntrakt, 215

Suspensio retropubica, 185, 324

Testiculo, tumores concordantes en gemelos, 353

Thyrocalcitonin, urinary calcium homeostasis, 285

Tuberous sclerosis, renal leiomyosarcoma, 406

Tumores renaies en la esclerosis cerebral tuberosa, 406

556

Index rerum

Twins, concordant tumors of the testicles, 353

Ulceras vesicales trás el Röntgenterapia,

429 Uraemia postoperatoria, 312 Urée du sang après des operations sur la

prostate, 312 Ureteral catheterization, 366 Ureterectasie congenitale, etude des

fibres nerveuses, 414 Uretero-intestinal implantation, sig-

moidal myotomia, 446 Uretère, operations plastiques, 425, 435 Uretersonden, Untersuchungen nach

längerer Liegedauer, 366 Urethra, fistulae and mural defects, 461 -, meatoplastia, 468 Uric acid calculi, 193

- $\quad$ - lithiasis, 193, 492, 506

lithiasis, prophylaxis by Allopurinol,

506 Urinary calculi, composition, 178

calcium homeostasis, Thyrocalcitonin, 285

fibrinolytic activity, 208

retention with herpes zoster, 222

tract, plastic surgery, 215 Urolithiasis, phosphate therapy, 483 Urolithiasis, Röntgenstruktur, Lósbar-

keit, 193 
Vesical neck, aponeurotic suspension, 185

neck, plasty and prostatectomy, 227

ulcer following X-irradiation, 429 Vesico-ureteral reflux in adults, 124 in children, 97, 141

Vessie, operations plastiques, 84

plastique, réabsorption des constitants urinaires, 435

rectale, 1, 29, 47

-, suspension aponévrotique du col, 185 -, ulcère radique, 429 Vejiga rectal, 1, 29, 47 -, suspension aponeurótica del cuello, 185

$\mathrm{X}$. Xanthogranulomatous pyelonephritis

68

Zwillinge

konkordante Hodentumoren

353 